\title{
Self-removing passive drain to facilitate post-operative care via telehealth during the COVID-19 pandemic.
}

\author{
Ryan K. Orosco, MD, FACS ${ }^{1}$ \\ ${ }^{1}$ UC San Diego Health, Moore's Cancer Center
}

April 28, 2020

\begin{abstract}
Background Telehealth post-operative visits are an attractive strategy to minimize exposure, especially during the SARS-CoV-2 (COVID-19) pandemic. The use of a surgical drain often prevents this minimal-exposure approach in that patients return to the outpatient clinic for drain removal. Methods and Results Following unilateral neck dissection, the customary closed-suction drain was replaced with a self-removing, passive drain dressing to facilitate same-day discharge and telehealth post-operative follow-up. The patient removed the dressing and drain at home during a telehealth visit on post-operative day four and she healed favorably without signs of infection or seroma. Conclusions When thoughtfully applied in the appropriate clinical context, small practice adaptations like this can facilitate telehealth solutions that diminish unnecessary exposure for patients, their caregivers, and healthcare staff.
\end{abstract}

Jesse R. Qualliotine, $\mathrm{MD}^{1}$ and Ryan K. Orosco, $\mathrm{MD}^{1,2 *}$

${ }^{1}$ Division of Otolaryngology-Head and Neck Surgery, Department of Surgery, University of California San Diego, La Jolla, CA, USA

${ }^{2}$ Moore's Cancer Center, University of California San Diego, La Jolla, CA, USA

*Corresponding Author: Ryan K. Orosco, MD, FACS

Assistant Professor, Head and Neck Surgical Oncology

UC San Diego Health, Moore's Cancer Center

3855 Health Sciences Dr, MC\#0987, La Jolla, CA 92093, United States

Telephone: 619-543-7895; Fax: 619-543-5521; Email: rorosco@health.ucsd.edu

Running Title: COVID neck drain

Keywords: COVID-19, Telemedicine, Neck Dissection, Head and Neck Neoplasms, Drainage

Conflict of Interest Statement:

None of the authors have published or submitted any related papers from the same study or have any conflicts of interest or financial disclosures to report.

\section{Abstract}

Background 
Telehealth post-operative visits are an attractive strategy to minimize exposure, especially during the SARSCoV-2 (COVID-19) pandemic. The use of a surgical drain often prevents this minimal-exposure approach in that patients return to the outpatient clinic for drain removal.

Methods and Results

Following unilateral neck dissection, the customary closed-suction drain was replaced with a self-removing, passive drain dressing to facilitate same-day discharge and telehealth post-operative follow-up. The patient removed the dressing and drain at home during a telehealth visit on post-operative day four and she healed favorably without signs of infection or seroma.

\section{Conclusions}

When thoughtfully applied in the appropriate clinical context, small practice adaptations like this can facilitate telehealth solutions that diminish unnecessary exposure for patients, their caregivers, and healthcare staff.

\section{Introduction}

The SARS-CoV-2 (COVID-19) pandemic is a rapidly developing challenge that affects all aspects of clinical care. As of April 11, 2020, the Centers for Disease Control and Prevention (CDC) reports 492,416 cases and 18,559 deaths from the United States alone. ${ }^{1}$ In order to increase the capacity of hospitals to care for the predicted volume of cases and decrease virus transmission rates, the US Center for Medicare and Medicaid Services issued recommendations to postpone all non-essential surgeries and procedures. ${ }^{2}$ To this end, many health systems and individual surgeons have adjusted their surgical caseloads appropriately and dramatically increased their utilization of telehealth encounters.

For many malignancies of the head and neck, a significant delay to treatment is known to be associated with worse overall survival, ${ }^{3}$ and such cases are proceeding to the extent that health systems can accommodate the case load. Statements published by AHNS leadership and others advocate that surgeries for cancer should be prioritized among others, especially when delay would adversely affect outcome. ${ }^{4,5}$ In this COVID era, telehealth post-operative visits are an attractive strategy to minimize exposure in a variety of circumstances including initial triage, counseling, and even post-operative settings. ${ }^{6}$ In the post-operative period, the use of a surgical drain often prevents this minimal-exposure approach in that patients return to the outpatient clinic for drain removal. Herein we present a case illustrating one solution using a self-removing, passive drain dressing after a neck dissection to facilitate same-day discharge and telehealth post-operative follow-up.

\section{Case Report}

A 57-year-old female presented in early March 2020 with a 2-month history of an enlarging $2 \mathrm{~cm}$ submandibular mass. She was otherwise asymptomatic and the remainder of her physical examination including flexible laryngoscopy was unremarkable. An ultrasound-guided fine needle biopsy demonstrated malignant adenoid cystic carcinoma and her staging CT had no evidence of cervical lymphadenopathy or pulmonary metastasis. In early April 2020 she underwent an uncomplicated ipsilateral neck dissection, levels I - III. Perioperative antibiotics were not administered for this clean site surgery. The neck was closed over a quarter-inch Penrose drain which was brought out through the posterior aspect of the incision. The remainder of the incision was closed with deep absorbable sutures and skin glue. The Penrose drain was sutured to gauze that was fashioned into a dressing with additional gauze and transparent film dressing (Tegaderm) in such a fashion that the drain would be pulled as the patient removed her dressing at home in several days. The inferior portion of the dressing was not sealed, and the patient was instructed to change the overlying gauze as needed for drainage (Figure 1). Detailed wound care instructions and return precautions were provided regarding seroma, hematoma, and wound infection. The patient was discharged to home on the day of surgery and remained in communication with the surgeon via phone. On post-operative day 4 , the patient removed the dressing and drain during a telehealth visit. The patient's incision was healing favorably without signs of 
infection or seroma. Maintaining close communication with the patient, she has done well without wound issues (Figure 2).

\section{Discussion}

Precautions spurred by COVID have led to an expansion of telehealth utilization. Video visits can effectively replace post-operative visits that do not require physical manipulation of the wound or dressings in order to eliminate non-essential contacts with the healthcare system and reduce virus transmission risk. If the visit cannot be performed remotely or postponed, donning of appropriate personal protective equipment (PPE) is crucial. ${ }^{7}$ Given the anatomic distribution of high viral load sites in the nasopharynx and mucosal airways, providers performing head and neck examinations appear to be at heightened risk of transmission.

In this case, to facilitate a telehealth post-operative visit a simple Penrose drain sutured to the overlying gauze dressing was used in lieu of the customary closed-suction drain. Although similar levels of evidence are not available for the lateral neck, in the post-thyroidectomy and central neck literature, randomized clinical trials and meta-analyses have demonstrated no statistically significant differences in rates of neck hematoma or seroma between patients with or without an active, closed-suction drain. ${ }^{8}$ Furthermore, uncomplicated seromas can be managed expectantly with needle aspiration in the rare cases that they do occur.

Even during a pandemic, patient safety is paramount and clinical judgement must be utilized when considering alterations to usual practices. The drain technique and post-operative follow-up method utilized in this case example must be thoughtfully implemented. Informed discussions and open lines of communication are paramount. Both patient and surgical factors should be considered. Alternative drainage methods are not well-suited for some patients and circumstances. Furthermore, traditional post-operative pathways should be followed for higher-risk procedures such as those with a heightened risk of salivary fistula and chyle leak. When thoughtfully applied in the appropriate clinical context, small practice adaptations like this can facilitate telehealth solutions that diminish unnecessary exposure for patients, their caregivers, and healthcare staff.

\section{References}

1. CDC. Coronavirus Disease 2019: Cases in U.S.

2. Non-Emergent, Elective Medical Services, and Treatment Recommendations. Centers for Medicare $\mathcal{E}$ Medicaid Services . 2020:Mar 18.

3. Murphy CT, Galloway TJ, Handorf EA, et al. Survival Impact of Increasing Time to Treatment Initiation for Patients With Head and Neck Cancer in the United States. J Clin Oncol . 2016;34:169-178.

4. How COVID-19 is Affecting our Head and Neck Community. Am Head Neck Soc From AHNS Leadersh AHNS Patient Care Div .:March 23, 2020.

5. Hanna T, Evans G, Booth C. Cancer, COVID-19 and the precautionary principle: prioritizing treatment during a global pandemic. Nat Rev Clin Oncol . 2020.

6. Rimmer RA, Christopher V, Falck A, et al. Telemedicine in Otolaryngology Outpatient Setting — Single Center Head and Neck Surgery Experience. Laryngoscope . 2018;128:2072-2075.

7. Chan J, Tsang R, Yeung K, et al. There is no routine head and neck exam during the COVID-19 pandemic. Authorea . 2020;1.

8. Sanabria A, AL C, Silver C, et al. Routine Drainage After Thyroid Surgery - A Meta-Analysis. J Surg Oncol . 2007;96:273-280.

Figure 1: A dressing was fashioned from gauze and transparent film dressing (Tegaderm) over a Penrose drain sutured to overlying gauze so that the drain would be pulled automatically as the patient removes the 
adherent dressing. The inferior aspect is left open to allow fluid egress and the patient was instructed to change the outer gauze as needed.

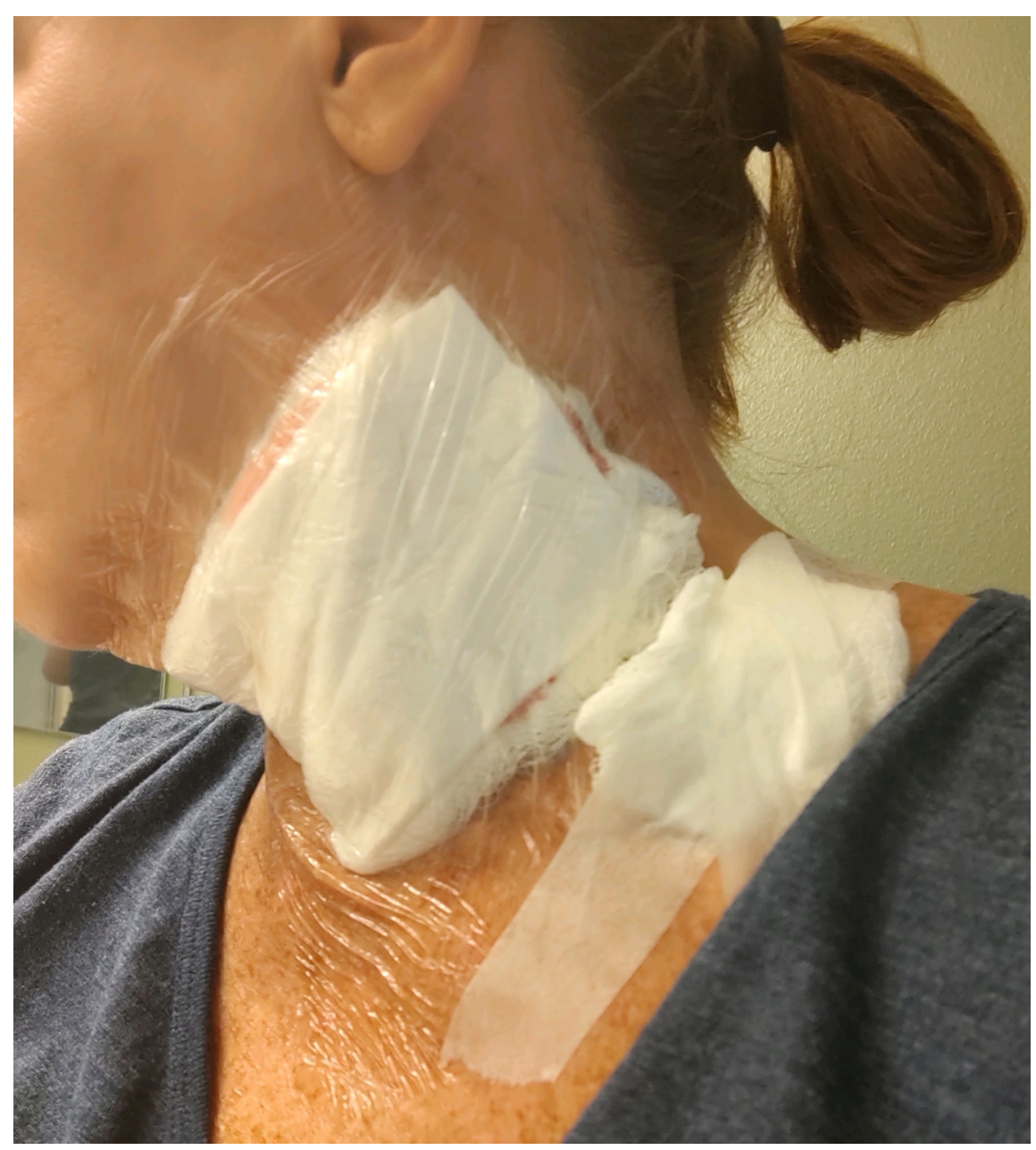

Figure 1: This is a caption

Figure 2: Healing incision following removal of the drain and dressing at home on post-operative day 7 . The neck was closed with deep absorbable suture and skin glue and this photo obtained from the telehealth encounter shows no evidence of seroma or surgical site infection. 


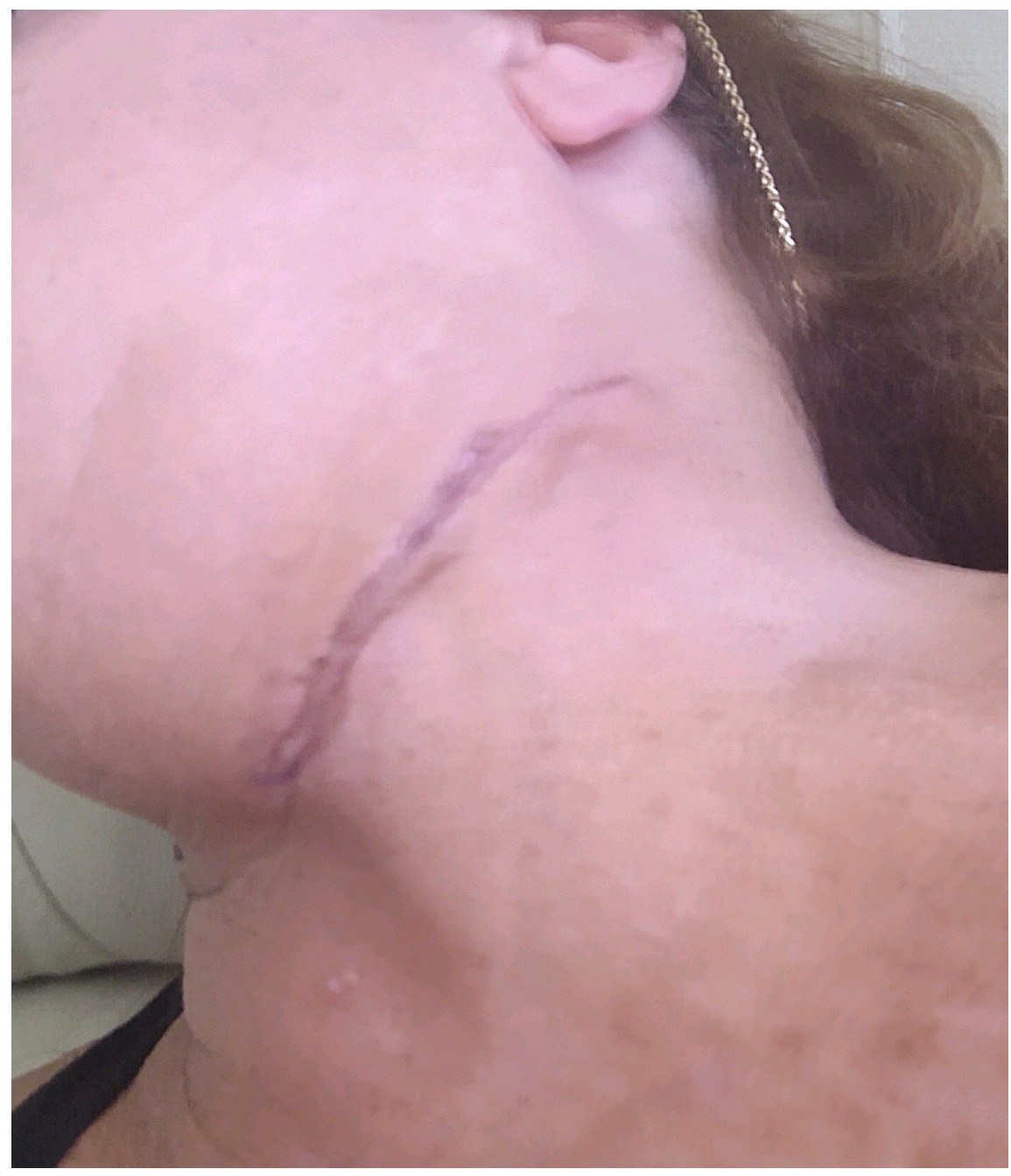

Figure 2: This is a caption 